\title{
Guía Práctica Clínica: Guía para el abordaje del paciente adulto con mareos (segunda parte: vértigo)
}

\author{
The adult dizzy patient (second part: vertigo)
}

Andrés Pichón Riviere* y Guillermo Videla**

\begin{abstract}
Resumen
Las causas periféricas de vértigo son las más frecuentes y comprenden el $85 \%$ de los casos. Se asocian a nauseas y vómitos, y son en su mayoría, autolimitadas y benignas. Los pacientes con vértigo central suele manifestar mayores trastornos en la deambulación y compromiso de otras estructuras del sistema nervioso central como ataxia, debilidad, disartria. Se recomienda chequear los signos vitales y hacer una evaluación neurológica, cardiológica y otológica a través de la anamnesis y el examen físico. Este artículo describe la clasificación sindromática de los pacientes con vértigo, las entidades clínicas más frecuentes, las herramientas diagnósticas de mayor utilidad, y el abordaje en atención primaria de los pacientes con vértigo periférico.
\end{abstract}

\begin{abstract}
The main causes of vertigo are peripheral in origin and comprise $85 \%$ of cases. They are associated with nausea and vomits and mostly self limited and benign. Patients with central vertigo usually show greater gait disturbances and other signs of central nervous system compromise such as ataxia, weakness or dysartria. A comprehensive evaluation of vital signs, as well as search for neuro, cardio and otological red flags through anamnesis and physical examination is recommended. This article describes the classification of patients suffering from vertigo, its most frequent clinical presentations, the most useful diagnostic tools, and primary care management of patients with peripheral vertigo.
\end{abstract}

Palabras clave: vertigo, evaluación, rehabilitación vestibular. Key words: vertigo, evaluation, vestibular rehabilitation.

Pichón Riviere A y Videla G. Guía para el abordaje del paciente adulto con mareos (segunda parte, vértigo). Evid. actual. práct. ambul; 10(3): 87-91, may-jun.2007.

\section{Introducción}

Una vez determinado que el paciente tiene vértigo -la percepción de que él gira o de que gira su ambiente 1 - es importante determinar si este es periférico o central. Aquellos trastornos que comprometan al nervio y/o al laberinto serán periféricos, y los que afectan al cerebelo o al tronco cerebral serán centrales (axiales) $)^{2}$.

Las causas periféricas de vértigo son las más frecuentes (85\%) en su mayoría, autolimitadas y benignas, aunque los síntomas como nauseas y vómitos suelen ser más severos. El vértigo central es menos frecuente (15\%) y los síntomas acompañantes son más leves; aunque suele haber compromiso de otras estructuras del sistema nervioso central (SNC) con diferentes manifestaciones y específicamente el trastorno en la deambulación es mayor. El sitio de la lesión suele identificarse por la presencia de síntomas asociados como por ejemplo, el compromiso auditivo (hipoacusia o acúfenos) al afectar el laberinto o el nervio vestíbulo coclear. También puede observarse una alteración a nivel del nervio facial cuando el trastorno esta ubicado en el canal auditivo interno. Los vértigos de origen central en los que se comprometen los núcleos vestibulares del tronco cerebral o el cerebelo y, por su estrecha vinculación anatómica con otras áreas neurológicas, virtualmente siempre presentan otra sintomatología neurológica asociada como ataxia, debilidad, disartria, etc. Ver cuadro 1.

Cuadro 1: características del vértigo periférico vs. central.

\begin{tabular}{l|l|l} 
& \multicolumn{1}{|c|}{ Periférico } & \multicolumn{1}{c}{ Central } \\
\hline Náuseas y Vomitos & Severos & Moderados \\
\hline Frecuencia & Más de $90 \%$ de los casos & Menos de 10\% de los casos \\
\hline Localización anatómica & $\begin{array}{l}\text { Oído interno, nervio vestibular } \\
\text { hasta el tronco cerebral }\end{array}$ & Tronco cerebral, cerebelo \\
\hline Síntomas asociados & Otológicos (acufenos, hi poacusia) & Otros síntomas neurológicos \\
\hline Deambulación & Posible & Muy comprometida \\
\hline Nistagmus & $\begin{array}{l}\text { Unidireccional rotatorio u } \\
\text { horizonto-rotatorio (no vertical) }\end{array}$ & $\begin{array}{l}\text { Cambiante. Puede ser } \\
\text { puramente vertical }\end{array}$ \\
\hline Duración & Corta & Larga \\
\hline Compensación central & Rapida y completa & Lenta y parcial \\
\hline
\end{tabular}

Etiología y presentación clínica de los diferentes tipos de vértigo

\section{Vértigo de origen central}

Es más común en pacientes ancianos, hipertensos, diabéticos o con enfermedad cardiovascular. Son causas de vértigo central la isquemia o infarto de tronco cerebral (enfermedad arterial vertebro basilar) las enfermedades desmielinizantes (esclerosis múltiple, desmielinización postinfecciosa, síndrome paraneoplásico) los tumores del ángulo pontocerebeloso (neurinoma del acústico, meningioma, colesteatoma, metástasis, etc.) las lesiones intrínsecas del tronco cerebral (tumor, malformaciones arteriovenosas) las convulsiones (muy raras) y las desórdenes heredo familiares (ej. ataxias espinocerebelosas).

Enfermedad arterial vértebro basilar: los episodios pueden ser causados por alteraciones en la circulación del circuito posterior que se encarga de irrigar al tronco cerebral donde se ubican los núcleos vestibulares. Estos pacientes suelen ser añosos, con múltiples factores de riesgo cardiovascular, pero también debemos pensarlo en jóvenes con antecedentes de traumatismo cefálico en los que se pudo haber producido una disección arterial cervical. Suele haber otros síntomas neurológicos acompañantes como pérdida de la visión, diplopía, parestesias periorales, ataxia, ataque de caída ("drop attack") disartria, etc. Si los síntomas son transitorios debe pensarse en un accidente isquémico transitorio (AIT) que duran un mínimo de cinco minutos. Alrededor de $25 \%$ de los pacientes con AIT vertebro basilar puede tener vértigo como manifestación inicial, al que se agregan luego otros síntomas relacionados con el compromiso del SNC.

Tumor del ángulo pontocerebeloso o neurinoma del acústico: desde el punto de vista anatómico es una causa periférica, pero habitualmente se la engloba dentro de las centrales ya que puede también comprometer al tronco cerebral por compresión. Originado en las células de Schwann de la rama vestibular del VIII par dentro del conducto auditivo interno, al crecer puede comprometer otras estructuras como la rama auditiva, el nervio facial (VII par) el trigémino (V) hasta el tronco cerebral y el cerebelo. Es muy raro que la manifestación ini-

*Médico Especialista en Medicina Familiar y Master en Ciencias Epidemiológicas. Director Ejecutivo del Instituto de Efectividad Clínica y Sanitaria. ** Médico Especialista en Neurología. Servicio de Neurología del Hospital Italiano de Buenos Aires. 
cial de este tumor sea la presencia de episodios aislados de vértigo. Los síntomas más comunes son una hipoacusia progresiva y acúfenos del lado afectado, inestabilidad en la mitad de los casos, encontrándose vértigo sólo en $20 \%$ de los pacientes. Es una causa poco frecuente de vértigo, pero debe pesquisarse ante una disminución asimétrica y progresiva de la audición ${ }^{3}$.

Esclerosis Múltiple: es una enfermedad de muy baja incidencia y menos de $10 \%$ de estos pacientes presentará vértigo. Cuando este es el síntoma inicial puede desconcertar al médico ya que simula una causa periférica. Su evolución en brotes y remisiones y la aparición de otra sintomatología como los trastornos de la motilidad ocular, problemas urinarios, déficits sensitivos o motores, ayudan a sospecharla.

\section{Vértigo de origen periférico}

El vértigo periférico es el tipo más común de vértigo. Debe sospecharse cuando el cuadro es de vértigo aislado o se acompaña sólo de síntomas auditivos, siendo el resto del examen neurológico normal. Las causas más frecuentes son la vestibulopatía periférica (laberintitis, neuronitis vestibular, vestibulopatía aguda y recurrente) el vértigo posicional paroxístico benigno, el vértigo postraumático, la vestibulotoxicidad (vértigo inducido por drogas) y la enfermedad de Ménière. Otras más raras son la infección bacteriana local, la cúpulo-litiasis, la otoesclerosis, la fístula laberíntica y el infarto laberíntico.

Vestibulopatía periférica, laberintitis, neuronitis vestibular o vestibulopatía periférica: todos estos nombres se refieren a una misma entidad de probable origen inflamatorio que afecta al laberinto o al nervio vestibular. Se caracteriza por el inicio brusco de un cuadro vertiginoso severo, generalmente un único episodio (raramente recurrente) que se acompaña de nauseas y vómitos. En el comienzo los síntomas son incapacitantes y puede requerir la internación del paciente, ya que estos pueden no tolerar la medicación ni la hidratación oral. La severidad suele disminuir en cuatro días. En el examen físico se evidencia nistagmus de tipo periférico. Suele existir el antecedente de un cuadro infeccioso de las vías aéreas superiores en los días previos al inicio del vértigo.

El paciente retornará gradualmente a su actividad normal y en la mayoría de los casos, con una recuperación total antes de los dos meses.

Un subtipo especial es el síndrome de Ramsay Hunt, causado por el virus herpes zoster que compromete los pares craneanos VII y VIII, que debe sospecharse cuando el vértigo agudo aparece en el contexto de dolor facial y/o auricular.

Vértigo posicional paroxístico benigno (VPPB): es la causa más frecuente de vértigo $(50 \%)^{4}$. Se da generalmente en pacientes mayores y es provocado por el desplazamiento anormal de cristales de carbonato de calcio dentro de los conductos semicirculares. Suele ser idiopático en más de la mitad de los casos, pero en el resto puede identificarse la causa del desprendimiento de los cristales (ej. traumatismo de cráneo leve).Existen distintos tipos de VPPB según el conducto semicircular afectado, siendo el más común el del canal semicircular posterior.

En este caso es típico que el paciente refiera presentar los síntomas al acostarse o lateralizarse en el decúbito y también en ciertas tareas domésticas como tender la ropa o colocar algo en una repisa alta (hiperextensión cefálica).

El vértigo suele durar menos de un minuto y puede estar asociado a nauseas, vómitos y diaforesis. Existe una relativa ausencia de síntomas durante la marcha, la sedestación o la bipedestación. El cuadro es reproducible en el consultorio mediante maniobras de desencadenamiento, con las que se obtiene un nistagmus con una latencia (tiempo a su inicio) menor a 40 segundos, agotable (dura menos de un minuto) fatigable (es menor a medida que se repite la maniobra) geotrópico (vertical hacia la tierra) y rotatorio.

Vértigo postraumático: un traumatismo craneano puede generar vértigo por múltiples mecanismos. Si el síntoma aparece inmediatamente luego del trauma, nos debe hacer sospechar un daño laberíntico. En ausencia de otras alteraciones en el examen deberíamos investigar una posible fractura del hueso temporal o una fístula perilinfática (conexión anormal entre el oído medio y el interno).

Otro mecanismo de daño del oído interno puede ser provocado por los cambios bruscos de presión (ej. buceo o paracaidismo) en los que se produce una ruptura traumática del tímpano con disminución de la audición, acúfenos y vértigo.

Lo más frecuente es que el vértigo aparezca unos días después del trauma, pudiendo ser producto de una hemorragia laberíntica o de un VPPB provocado por el desprendimiento de cristales por el golpe. En estas situaciones no existe un daño severo del laberinto y el pronóstico es benigno.

Enfermedad de Ménière: suele aparecer durante la quinta década de la vida. La tríada sintomatológica esta compuesta por vértigo, acúfenos y pérdida auditiva fluctuante que evoluciona con los años a una hipoacusia irreversible. En la mayoría de los pacientes el cuadro es unilateral aunque en $5 \%$, puede tornarse bilateral llevando a una sordera. Generalmente, las crisis comienzan de manera espontánea con una sensación de plenitud auditiva, con disminución de la audición y acúfenos unilaterales, apareciendo luego un severo vértigo con nauseas y vómitos.

Este llega en pocos minutos a su pico máximo y luego disminuye lentamente de intensidad para desaparecer al cabo de varias horas, pudiendo quedar una sensación de embotamiento durante varios días luego de la crisis. La teoría fisiopatogénica más aceptada es la del hidrops endolinfático y estaría relacionada con un aumento de la presión endolinfática dentro del oído interno que produciría excitación y daño de las células ciliadas vestibulares y cocleares. El pronóstico es variable y puede evolucionar con crisis espaciadas por años sin afectación de la calidad de vida o hasta cuadros severísimos que requieren tratamiento quirúrgico.

Toxicidad por drogas: si bien las drogas vestibulotóxicas no producen generalmente vértigo, sí dan inestabilidad, mareos y oscilopsia (ilusión de movimiento del campo visual) por la afectación bilateral que producen.

Hay que destacar la acción de los aminoglucósidos (especialmente la estreptomicina y la gentamicina que dañan las células pilosas de los receptores vestibulares) y algunos agentes quimioterápicos (cisplatino). Estos fármacos se concentran en la endo y perilinfa por lo que hay que ser cuidadoso en su indicación, más aún si el paciente tiene trastornos renales.

Cinetosis: no es propiamente un tipo de vértigo, pero dada su alta frecuencia es importante saber en que consiste y como tratarlo. Se da en personas con una mayor sensibilidad a la estimulación vestibular, que se produce generalmente durante viajes en barco, auto, colectivo y avión.

Se debería a una confrontación entre la información que recibe el cerebro del sistema vestibular que indica movimiento y la visual más la propioceptiva, que indican quietud. 


\section{Sistemática de estudio}

Interrogatorio

A un $72 \%$ de los pacientes mareados se los puede diagnosticar sólo con el interrogatorio. Si el paciente no logra precisarnos bien su sintomatología, el interrogatorio dirigido nos ayudará. La descripción del primer episodio o del inicio del cuadro y si este ha remitido o es progresivo, es de gran importancia, ya que muchas veces el trastorno actual ha diferido del problema de inicio. La duración de los episodios nos dará claves acerca de su etiología. Si son breves (segundos a algunos minutos) nos orientaremos hacia VPPB; si duran minutos a horas pensaremos en AIT, enfermedad de Ménière, migraña-vértigo, etc.; y si duran varios días, en migraña-vértigo, tumor cerebral, ACV, neuronitis vestibular, laberintitis, ototóxicidad, etc.

Los factores desencadenantes o atenuantes siempre deben tenerse en cuenta: posición cefálica en la cual aparecen (VPPB) o al levantarse bruscamente de la cama (hipotensión ortostática).

Deben anotarse los antecedentes del paciente: antecedentes familiares de cuadros similares (heredoataxias) enfermedades cardiovasculares, neurológicas, otológicas y exposición a fármacos ototóxicos, vasopresores, antidepresivos o anticonvulsivantes, que pueden ser el origen del problema y de resolución relativamente sencilla.

Indagaremos por la presencia de síntomas asociados que puedan indicar la localización anatómica del problema:

Otológicos: la presencia de hipoacusia unilateral y/o acúfenos nos indicará un posible compromiso vestibulococlear o del VII par. Si el trastorno es bilateral pensaremos en una etiología inmunológica o tóxica.

Neurológicos: ataxia, disartria o diplopía hablan de compromiso del tronco cerebral y sus estructuras vecinas (fosa posterior del encéfalo). Un compromiso del V y VII par nos habla de una alteración a nivel del conducto auditivo interno.

Cardiovasculares: palpitaciones, dolor precordial, disnea, sensación de pérdida de conocimiento o desmayos. Oscurecimiento de la visión al levantarse bruscamente (ortostatismo).

\section{Examen físico}

Comenzar por los signos vitales. La hipertensión arterial puede indicar compromiso asociado cerebrovascular y la hipotensión puede deberse a un trastorno secundario a algún fármaco o por un bajo gasto cardíaco. Controlarla en decúbito y de pie para evaluar posible ortostatismo (caída de la sistólica $20 \mathrm{mmHg}$ o más, tras estar de pie durante dos minutos). Taquicardias y bradicardias pueden relacionarse con síncope y mareos, mientras que un aumento del ritmo respiratorio se observa en las crisis de pánico. El aumento de la temperatura sugiere infección sistémica, otológica o del SNC.

Mirar el conducto auditivo externo buscando vesículas (síndrome de Ramsay Hunt) cerumen, rotura timpánica u oído medio ocupado (otitis media, colesteatoma, tumor del glomus, etc.). Examinar la audición con un diapasón o mediante el cuchicheo o la frotación del pelo cerca del pabellón auricular. Observar los reflejos pupilares y los movimientos oculares, pesquisando nistagmus (oscilación ocular involuntaria) que consta de dos componentes, uno lento y uno rápido.

Este último califica al nistagmus (ej. nistagmus derecho, izquierdo, etc). Evaluar los cambios que se producen en el nistagmus ante la fijación, posición ocular y posición cefálica, clasificándolo en espontáneo, evocado por la mirada (aparece con determinados movimientos oculares) y/o posicional (se desencadena a partir de ciertas posiciones cefálicas, por ejemplo el nistagmus del VPPB.

El nistagmus espontáneo puede ser de origen central o periférico (cuadro 2) y se evidencia cuando el paciente dirige su mirada hacia el frente, arriba, abajo y a ambos costados, pero sin desviar la misma más de 30 grados de la línea media. La presencia de nistagmus espontáneo debe considerarse patológica. Es importante solicitarle al paciente que realice los movimientos oculares espontáneamente, ya que si los hace siguiendo nuestro dedo puede suprimirse el nistagmus por la fijación.

También se puede tratar de observar los movimientos oculares del paciente con sus ojos cerrados, o sea a través de los párpados. La presencia de nistagmus en mirada extrema suele carecer de significado patológico.

En el vértigo periférico hay nistagmus espontáneo que mantiene su dirección aún con los movimientos oculares. Cuando el trastorno es central el nistagmus espontáneo cambia de dirección con cada movimiento ocular. Una excepción a esto es la presencia de nistagmus vertical puro no fatigable, que suele asociarse con un compromiso central.

Cuadro 2: diferencias entre nistagmus espontáneo periférico y central.

\begin{tabular}{l|l|l|l|l} 
& \multicolumn{1}{|c|}{ Apariencia } & \multicolumn{1}{|c|}{ Fjjación } & \multicolumn{1}{c|}{ Mirada } & \multicolumn{1}{c}{ Localización } \\
\hline Periférico & $\begin{array}{l}\text { Combinada torsional- } \\
\text { horizontal }\end{array}$ & Lo Inhibe & Unidireccional & $\begin{array}{l}\text { Laberinto 0 nervio } \\
\text { vestibular }\end{array}$ \\
\hline Central & $\begin{array}{l}\text { Pura vertical, } \\
\text { horizontal 0 torsional }\end{array}$ & $\begin{array}{l}\text { Poca 0 nula } \\
\text { inhibición }\end{array}$ & Cambiante & SNC, tronco 0 cerebelo \\
\hline
\end{tabular}

\section{Examen neurológico}

Evaluar la marcha y examinar los pares craneanos, la motilidad general, la fuerza, la sensibilidad y la coordinación.

Los reflejos vestibuloespinales mediante la prueba de confrontación de índices, la de Romberg y el Romberg sensibilizado con los pies en tandem (suele observarse una tendencia a caer en la dirección del componente lento del nistagmus). EI test de Unterberger cumple la misma función (se solicita al paciente que marche en el lugar con los ojos cerrados y puede observarse que rota hacia el oído hipovalente).

El test de Halmagyi o de sacudida cefálica brusca se utiliza para evaluar los reflejos vestibulooculares y consiste en provocar un pequeño y rápido movimiento cefálico horizontal luego de haberle solicitado al paciente que fije su mirada en la nariz del examinador. Normalmente los ojos permanecerán fijos en el punto indicado, en el caso de hipofunción vestibular se observará que sufren un desplazamiento en la dirección del movimiento que es corregido inmediatamente con un movimiento sacádico rápido.

Siempre debe efectuarse la maniobra de Dix-Hallpike ${ }^{5}$ o de Nylen-Barany (figura 1) primero hacia un lado y luego hacia el otro con el fin de chequear el oído interno opuesto. La aparición luego de una breve latencia de un nistagmus geotrópico (hacia el suelo) rotatorio, agotable y fatigable confirman el diagnóstico de VPPB del canal semicircular posterior del oído evaluado. 
Figura 1. maniobra de Dix-Hallpike o de Nylen-Bárány o Bárány.

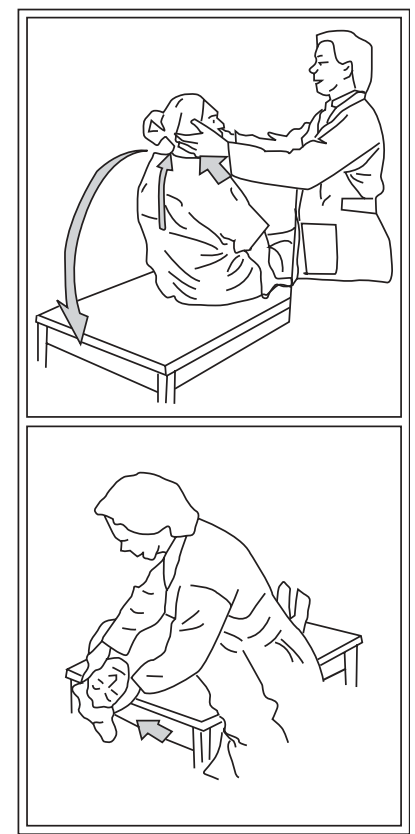

a) sentar al paciente en la camilla de manera que, al acostarlo, su cabeza cuelgue fuera del borde de la misma. Con el paciente sentado, rotarle la cabeza $45^{\circ}$ y pedirle que mire siempre hacia adelante sin fijar la mirada en ningún objeto y que no cierre los ojos ya que buscaremos la respuesta nistágmica en ellos

b) recostar rápidamente al paciente manteniendo la cabeza rotada y que esta cuelgue $30^{\circ}$ por debajo de la línea de la camilla.

c) Observarlo durante por lo menos 30 segundos. Debe evaluarse el tiempo que tarda en aparecer el nistagmus, su dirección y duración. También es importante considerar si aparecen síntomas y si el nistagmus tiende a desaparecer al repetir la maniobra (fatigabilidad).

\section{Tratamiento}

Vertigo paroxístico posicional benigno

EI VPPB cuenta con un tratamiento específico que consiste en maniobras de reposicionamiento (de Epley ${ }^{6}$ ) de los cristales del oído interno, que hace que los cristales anormalmente ubicados en el canal semicircular se desplacen hacia el utrículo solucionando definitivamente el problema (ver figura 2). El $80 \%$ de los pacientes se cura con sólo una maniobra y se llega a casi $100 \%$ de éxito si ésta se repite tres veces.

Ningún fármaco es efectivo para el tratamiento de este problema. Se recomienda sugerirle al paciente que tome un comprimido de 50mg de dimenhidrato dos horas antes de realizar las maniobras, ya que éstas generalmente desencadenan un vértigo severo con náuseas y vómitos.

\section{Laberintitis}

Al inicio del cuadro, el tratamiento sintomático mediante antieméticos y antivertiginosos resulta útil para mejorar al paciente. Su duración de éste debe estar controlada por el médico ya que los medicamentos antivertiginosos interfieren con la recuperación de la función vestibular. Ni bien el paciente comienza a deambular y ceden los vómitos, se debe indicar un esquema de rehabilitación vestibular.

\section{Enfermedad de Ménière}

Existe un tratamiento sintomático durante el ataque, similar para todo síndrome vertiginoso agudo que se explicará más adelante. Entre las crisis pueden utilizarse diuréticos (hidroclorotiazida) o betahistina. También se recomienda una dieta baja en sodio y evitar excitantes como las xantinas.

\section{Otras causas}

Ante toxicidad por drogas debe suspenderse el fármaco siempre que sea posible, luego evaluar el grado de afectación del paciente y comenzar con rehabilitación vestibular. A los pacientes que sufren cinetosis se les puede dar algunos consejos para prevenir o aminorar los síntomas como alinear la cabeza y el cuerpo con la dirección del movimiento (ej. viajar mirando hacia adelante) obtener una visión estable del horizonte real y controlar la respiración, manteniendo un ritmo regular y relajado. De no ser efectivas estas medidas se pueden utilizar antivertiginosos pero recordando siempre que deben ser ingeridos una hora antes de la exposición al estímulo. Si e estímulo va a durar días (ej. un viaje en barco) no es necesario mantener el tratamiento ya que los síntomas suelen remitir con la habituación.

Si se sospecha un neurinoma del acústico debe consultarse al especialista, quien indicará estudios audiológicos y por imágenes. El tratamiento suele ser quirúrgico.

Ante isquemia o infarto del tronco cerebral se procede de manera similar al enfoque de todo paciente con AIT o ACV. Sabiendo cual fue el origen del trastorno (ateromatosis, enfermedad de pequeños vasos, cadioembolismo, embolismo arterio-arterial, disección arterial, etc.) se tratará con control estricto de los factores de riesgo cardiovascular asumiendo que se trata de un paciente con enfermedad cardiovascular instalada (antiagregación, metas estrictas de lípidos y tensión arterial) eventual anticoagulación y en algunos casos, mediante abordaje endovascular o quirúrgico.

\section{Tratamiento farmacológico del vértigo}

El fármaco ideal para el tratamiento del vértigo debería suprimir totalmente el vértigo, las nauseas y los vómitos; ayudar a una mayor y más rápida compensación de los síntomas; no poseer efectos adversos y evitar las recurrencias, pero lamentablemente este medicamento no existe ${ }^{7}$. La batería farmacológica de hoy cuenta con dos tipos principales de drogas: los supresores vestibulares y los antieméticos, que pueden indicarse por vía oral si no hay vómitos, o en su defecto por vía rectal, sublingual o parenteral.

Los supresores vestibulares sólo son efectivos para causas vestibulares y también presentan cierta acción antiemética. Estos fármacos afectan la compensación central, por lo que un uso prolongado e innecesario repercutirá negativamente en el pronóstico del paciente. Además, un efecto adverso común de todo el grupo es el de provocar sedación, por lo que puede aumentar significativamente el riesgo de caída de nuestro ya inestable paciente. A mayor efecto antivertiginoso mayor sedación. Para cuadros severos como el estadío inicial de una laberintitis, algunas crisis de Ménière o el infarto laberíntico deberemos usar una medicación potente como prometacina intramuscular a una dosis de $12,5 \mathrm{mg}$ cada seis u ocho horas, que tiene también acción antiemética; pudiendo ser de utilidad el lorezepam sublingual. Para cuadros moderados con buena tolerancia oral se utilizan medicamentos como el clonazepam oral o sublingual ( 0,5 a $2 \mathrm{mg}$ cada seis $\mathrm{u}$ ocho horas) el dimenhidrato oral (50mg cada cuatro a seis horas) o la meclizina oral (25 a $50 \mathrm{mg}$ cada cuatro a seis horas). Las benzodiazepinas, además de su efecto antivertiginoso, pueden ser útiles por su acción ansiolítica.

Entre los antieméticos destacamos la metoclopramida que puede administrarse por vía sublingual, endovenosa u oral y es buena opción para casos moderados. Para los severos con vómitos incoercibles, algunos antieméticos utilizados para 
combatir las náuseas asociadas a quimioterapia como el ondasentron o granisetron son muy efectivos y se administran por vía parenteral.

\section{Rehabilitación vestibular}

Consiste en un programa de ejercicios cuyo objetivo principal esta dirigido a mejorar el trastorno del equilibrio, disminuir el riesgo de caída, eliminar el vértigo y aumentar la funcionalidad global del paciente. La rehabilitación vestibular ${ }^{9}$ se basa en la confrontación del paciente con estímulos que le desencadenan mareos y vértigo; lo que promueve una compensación del sistema nervioso central para conseguir así, nuevamente balancear el funcionamiento entre ambos oídos internos ${ }^{10}$. Existen algunos protocolos que pueden ser implementados por personal de enfermería ${ }^{11}$.

Figura 2: primer paso de la maniobra de Epley para reposicionar los cristales del oído interno.

Esta maniobra de reposicionamiento se inicia igual a la de DixHallpike pero una vez en el decúbito (a), se rota al paciente $45^{\circ}$ hacia el oído contralateral (b).

Luego se gira horizontalmente la cabeza y el cuerpo $135^{\circ}$ (c) y, finalmente, manteniendo la cabeza girada se sienta al paciente y se baja la barbilla unos $20^{\circ}$ respecto a la horizontal (d).
Es conveniente repetir dos o tres veces esta maniobra para lograr una mayor efectividad.

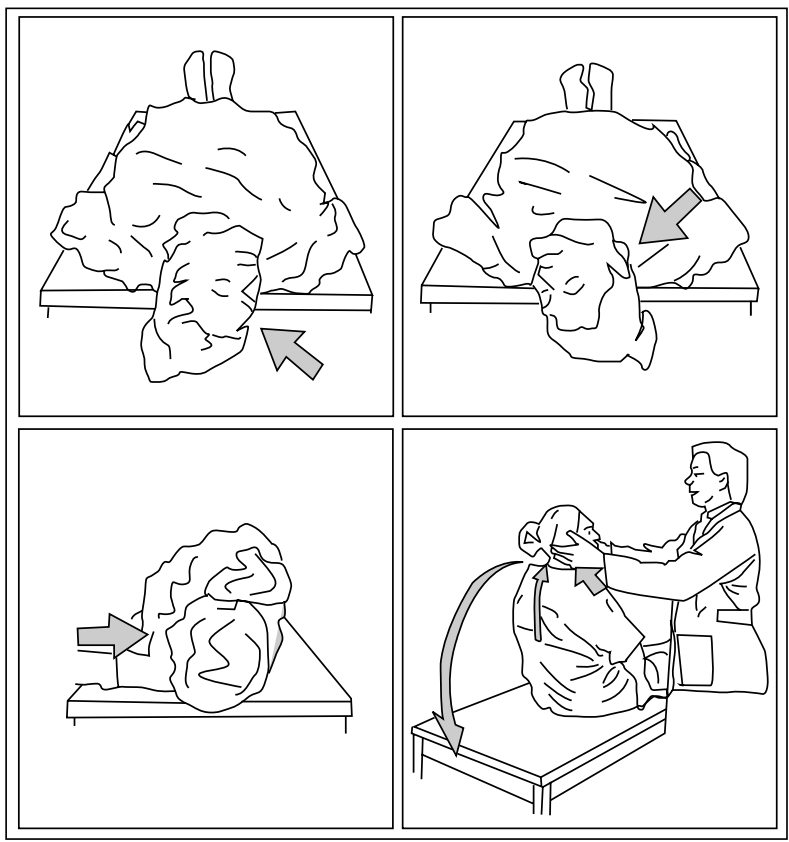

Recibido el 20/04/07 y aceptado el 23/05/07

\section{Referencias}

1. Lempert T. Vertigo. Curr Opin Neurol 1998. Feb; 11(1): 5-9. (Review).

2. Baloh R, Honrubia V. Clinical Neurophysiology of the vestibular system. 3rd De. Oxford, 2001

3. Gizzi M, et al. The diagnostic value of imaging the patient with dizziness. A Bayesian approach. Arch Neurol 1996; 53(12): 1299-304

4. Furman JM, Cass SP. Benign paroxysmal positional vertigo. N Engl J Med. 1999 Nov 18;341(21):1590-6. http://content.nejm.org/cgi/content/full/341/21/1590?ck=nck

5. Dix MR, Hallpike CS. The pathology, symptomology and diagnosis of certain common disorders of the vestibular system. Ann Otol Rhinol Laryngol 1952; $61: 987-1016$

6. Epley JM. The canalith repositioning procedure: for treatment of benign paroxysmal positional vertigo. Otolaryngol Head Neck Surg 1992 Sep; $107(3)$ : $399-404$.

7. Darlington CL, Smith PF. Drug treatment for vertigo and dizziness. N Z Med J 1998 Sep 11; 111 (1073): 332-334

8. Yardley L, et al. A randomized controlled trial of exercise therapy for dizziness and vertigo in primary care. Br J Gen Pract 1998; 48(429): 1136-40.

9. Smith T. La rehabilitación vestibular es una terapia efectiva para los mareos crónicos en atención primaria. Evid. actual. práct. ambul. 2005;8:72. Comentado de: Yardley L, Donovan-Hall M. et al. Effectiveness of primary care-based vestibular rehabilitation for chronic dizziness. Ann Intern Med. 2004;141: 598-605. PMID: 15492339 http://www.foroaps.org/hitalba-pagina-articulo.php?cod_producto=1661

10. Horak FB, et al. Effects of vestibular rehabilitation on dizziness and imbalance. Otolaryngol Head Neck Surg 1992 Feb; 106(2): 175-180.

11. Fruncillo M. tratamiento del vértigo posicional. Evid. act. pract. ambul. 9 (1):14. En-Feb.2006. http://www.foroaps.org/files/vretigo\%20dibujito.pdf 